\title{
Phonon band structure and electron-phonon interactions in metallic nanowires
}

\author{
Matthieu J. Verstraete ${ }^{1,2,3 *}$ and Xavier Gonze ${ }^{1,3}$ \\ 1 Unité PCPM and CERMIN, Université Catholique de Louvain, \\ Croix du Sud 1, B-1348 Louvain-la-Neuve, Belgium \\ 2 Physics Dept. University of York, Heslington, YO10 5DD, United Kingdom \\ 3 European Theoretical Spectroscopical Facility (ETSF)
}

\begin{abstract}
We present first-principles calculations of the phonon band structure and electron-phonon coupling in thin metallic nanowires. A full Brillouin zone analysis of the phonons is mandatory for the investigation of the nanowire structural stability: all the examined unstrained nanowires show instabilities whose wavevectors are located off the zone center. The unstable phonon modes are transverse, leading to a transition without a gap opening, in contrast with the usual Peierls distortion picture. Electron-phonon coupling yields orders-of-magnitude changes depending on the nanowire structure.
\end{abstract}

PACS numbers: 63.20.-e,71.15.Mb,73.21.Hb,73.22.-f

Metallic nanowires consisting of a single chain of evenly spaced atoms (monowires) have long been a theorist's playground : they have inspired many model systems for electrons, phonons and their coupling. One dimensional (1D) metallic systems were shown to be unstable against longitudinal distortion (i.e. pairing) by Fröhlich[1] and Peierls[2] in the 50s. This is, however, a simplified situation, and more general distortions must be considered. Batra [3] showed that transverse distortions can give rise to a different type of transition, which does not open a gap around the Fermi level (a phenomenon he coined Gapless Peierls Transition - GPT). The consequences of electron-phonon coupling (EPC) in monowires have been the focus of recent studies, e.g. [4], in particular to determine the effect of phonons on conductivity. Peierls transitions have been seen in first-principles simulations of carbon nanotubes[5, 6], and the issue of whether superconductivity can survive in 1D, despite Peierls transitions, is an open question. For both normal and superconductivity in 1D, understanding the interplay between structure, stability, and metallicity is essential for nanotechnological applications.

Nanowires or nanotubes with diameters superior to a few nanometers are produced nowadays for many different atomic species. In the past decade, it has also become possible to experimentally create and examine wires comprising just a few or even one strand of atoms. The most impressive success has been with gold[7, 8], but other metals have also been used in different experimental setups $[9,10]$. The potential applications of thin nanowires include integrating electronics on a molecular scale, improving scanning microscope tips, and increasing data storage densities if the wires are magnetic.

In the first thin gold nanowires visualized with an electron microscope[7], a large distance was observed between neighboring atoms along the wire. This distance was explained using first-principles calculations[11] to be an average distance between second neighbors in a zigzag (ZZ) structure, obtained by a transverse period-doubling of the monowire. Structures with small angles $\left(\simeq 60^{\circ}\right)$ between successive bonds have been found theoretically for many metals. Analogous ZZ structures, with an angle larger than 120 degrees, were proposed to be stable only for some metals (see e.g. Ref. 12).

Although these striking examples point to the need to examine critically the stability of thin $1 \mathrm{D}$ nanowires, general distortions (as examined in a 1D phonon band structure) beyond the center and edges of the the Brillouin zone (BZ) have not been considered. As we shall see below, the phonon structure for thin nanowires is very complex, and unstable modes can appear everywhere in the BZ. It is thus essential to consider the full phonon band structure, in order to present credible models to compare to experiment.

In the following, we present our calculational methods, then describe the electron and phonon band structures of several nanowires, made out of aluminum and lead. We do not consider gold here because bulk Au has almost no superconductivity to speak of, and it would be difficult to extricate the $1 \mathrm{D}$ effects from the intrinsic ones due to chemical nature. $\mathrm{Al}$ and $\mathrm{Pb}$ are representative of materials with weak (resp. strong) electron-phonon coupling in the bulk, and with three (resp. four) valence electrons per atom. Moreover, lead exhibits strong spinorbit (SO) coupling, with a remarkable influence on the electron band structure. We also consider the effect of tension in the case of $\mathrm{Al}$ : the $\mathrm{Al} \mathrm{ZZ} \mathrm{obtuse} \mathrm{wire} \mathrm{becomes}$ stable under tension. After the analysis of the electron and phonon band structures, the EPC is characterized. We observe variations of several orders of magnitude.

A number of experimental and theoretical works have appeared on the geometry[13], electronic structure[14], Peierls instabilities[15], and conductance[16] of $\mathrm{Al}$ monowires. To our knowledge, although $\mathrm{Pb}$ nanowires a few dozen nanometers in diameter can be produced[17], sub-nanometer $\mathrm{Pb}$ monowires have not been synthesized or studied theoretically.

Our calculations are performed using the ABINIT[18] 

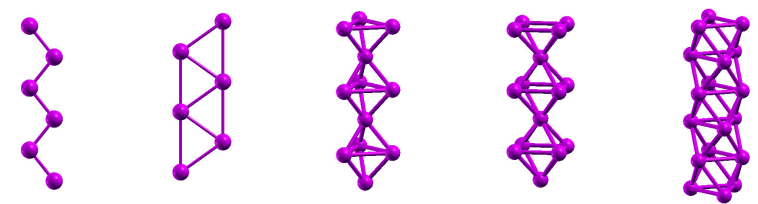

FIG. 1: Atomic structures of the zigzag obtuse and acute, and thicker 1-3, 1-4, and 3-3 nanowires.

density-functional theory[19, 20] package. We fully relax atomic forces (less than $5 \cdot 10^{-5} \mathrm{Ha} / \mathrm{bohr}$ ) and unit cell stresses along the wire (less than $1.5 \cdot 10^{-7} \mathrm{Ha} / \mathrm{bohr}^{3}$ ). A lateral cell size of 30 bohr gives converged forces and stresses. A plane-wave kinetic energy cutoff of $8 \mathrm{Ha}$ and a Troullier-Martins[21] pseudopotential are employed for $\mathrm{Al}$. For $\mathrm{Pb}$, we use a cutoff of $16 \mathrm{Ha}$ and a Hartwigsen-Goedecker-Hutter[22] pseudopotential, which accounts for SO coupling. We will employ the local density approximation[20]. Using gradient approximations should not influence the tendencies we will expose. The phonon properties are calculated through densityfunctional perturbation theory[23]. SO effects are treated through the pseudopotential (Ref. [22]). The SO operator is not considered explicitly; levels resulting from the pseudopotential are split due to the SO interaction with the core electrons. The implementation of the perturbation treatment will be presented elsewhere. We will report the (imaginary) frequencies of unstable phonon modes by the negative of their modulus. We employ 200 wavevectors in the full BZ of the single atom unit cells, and 100 for the others. The phonon quantities are sampled by 10 wavevectors in the full BZ. EPC quantities (i.e. Eliashberg $\alpha^{2} F$ functions) are calculated in a standard way $[24,25]$. As the wires are metallic, a Gaussian smearing of width $0.001 \mathrm{Ha}$ is used to improve the convergence with respect to the number of kpoints. The consequences of temperature are discussed below.

We consider different structural models for nanowires, shown in Fig. 1. The simple monowire, denoted 1-1, can present two types of transverse deformations, characterized by an obtuse $\left(>120^{\circ}\right)$ and an acute $\left(\simeq 60^{\circ}\right)$ angle between successive bonds. We call these structures the zigzag obtuse and acute wires. For both $\mathrm{Al}$ and $\mathrm{Pb}$ we find that the monowires spontaneously distort, if we allow the symmetry to be broken, with transverse displacements of the atoms, and relax to the obtuse ZZ structure. However, this configuration is only metastable: the $\mathrm{ZZ}$ acute wire, with an angle of about $60^{\circ}$ between successive bonds, has even lower energy. Longitudinal dimerization does not occur for any of the wires examined, in agreement with Ref. [12] and [14] for Al. We have also considered thicker wires in the case of $\mathrm{Al}$, constructed from FCC packing of atoms along the (111) and (100) directions. These wires will be called 1-3 and 3-3 in the former case, and 1-4 in the latter, depending on the num- ber of atoms in successive cross-sections of the wire (see Fig. 1). In each case, we fully relax the wire, within the available configuration space compatible with the overall symmetry.
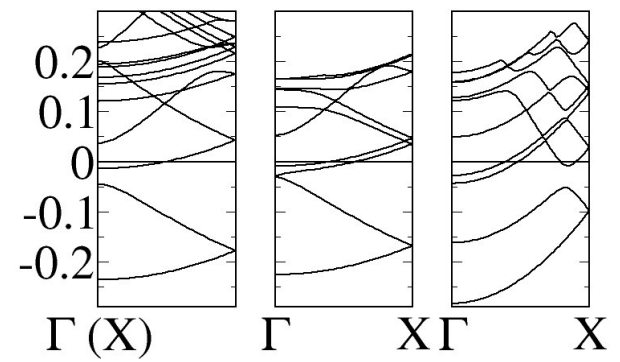

FIG. 2: Electron BS of $\mathrm{Al}$ nanowires (Ha). Left to right: 11, zigzag obtuse, and acute wires. The 1-1 bands have been folded back into half of the BZ for comparison with the zigzag cases.
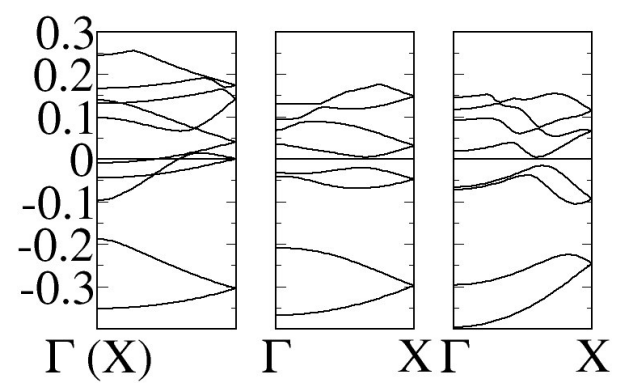

FIG. 3: Electron $\mathrm{BS}$ of $\mathrm{Pb}$ monowires (Ha). Left to right: 1-1, zigzag obtuse, and acute wires. The 1-1 bands have been folded back into half of the BZ for comparison with the zigzag cases.

The electronic band structure (BS) for the 1-1 and ZZ nanowires is shown in Fig. 2 for aluminum, and for lead in Fig. 3. Note the lack of a gap in the aluminum case, contrasting with the gap opening for the $\mathrm{ZZ}$ lead wire. If the SO interaction is removed, the gap closes again in lead nanowires.

The preservation of metallicity is very important in the GPT[3]. It is related to the presence of a mirror symmetry perpendicular to the wire axis, conserved by the transverse distortion but not the longitudinal one. In this respect, it is important to clarify the $\mathrm{Pb}$ case. We examine the symmetry of the ground state orbitals of the $\mathrm{ZZ} \mathrm{Pb}$ wire near the Fermi point. In the case without SO coupling the $\sigma$-like and $\pi$-like (i.e. symmetrical or with nodal plane in the plane of the wire) characters are preserved for the bands crossing at the Fermi level. Including SO coupling, the states above and below the $E_{F}$ have similar shape and neither a nodal plane nor a plane of symmetry. Thus, the SO coupling can mix the characters of the bands near the Fermi level, forcing them to anticross. The analysis of EPC in the $\mathrm{Pb}$ wire without SO coupling shows that it is only slightly smaller than with SO coupling, confirming the purely electronic explanation of the opening of a gap. The important point is that the bands cross near the Fermi level and that the 
anticrossing is sufficient to open a gap around $E_{F}$.

We now turn to the phonons. In $1 \mathrm{D}$ systems without cylindrical symmetry (i.e. excluding monowires), there is an additional zero-frequency mode at $\Gamma$ : beyond the three acoustic modes related to translational symmetry, a fourth mode is related to the rotational invariance around the wire axis. The supercell shape and the real-space integration grid in our calculations break this rotational invariance, but the effect is negligible for the cell sizes considered: this mode has a frequency of less than $1 \mathrm{~cm}^{-1}$ at $\Gamma$. For off-center wavevectors, the rotational mode becomes a twisting mode, and makes helix-like structures out of the $\mathrm{ZZ}$ monowires.

The phonon band structures of six nanowires are shown in Fig. 4 and 5. We have also computed the phonon band structures of the 1-3, 1-4 and 3-3 Al nanowires (not shown). Note that the aluminum ZZ nanowires $\left(2^{\text {nd }}\right.$ and $3^{\text {rd }}$ in Fig. 4) have been stretched. In all nine cases an instability develops for the unstrained nanowire: several transverse modes are unstable in most of the BZ, but longitudinal instabilities do not occur. For finite wires the phonons will sample the full BZ, and may also have unstable modes.

The Al ZZ obtuse nanowire can be made stable by increasing the unit cell length by $2 \%$ (the atom positions are relaxed), as shown in the middle of Fig. 4. Under the imposed tension all the phonon modes have positive frequencies. This is quite important as, experimentally, most thin nanowires are created under tension: we show here theoretically that simple, regular, ZZ structures can be stabilized under tension, but likely not without it. For the acute case, increasing the lattice spacing by up to $10 \%$ is still not enough to stabilize all of the phonons (Fig. 4 right): those at the $\mathrm{X}$ point become positive, but other modes in the middle of the BZ remain highly unstable. The ideal strengths calculated in Ref. [12] show that both $\mathrm{ZZ}$ structures for $\mathrm{Al}$ will break instead of relaxing to the next stable structure (ZZ acute to obtuse to linear chain). However, we see here that a truly stable structure does exist for the ZZ obtuse wire under tension.

The unit cells resulting from small $\vec{q}$ instabilities are very large. We have not searched for the final stabilized configurations of the different nanowires, that are likely complex structures arising from several transitions. The unstable modes of the monowires do not all disappear upon transverse distortion (with doubling of the cell), but the modulus of their frequencies is smaller. For the $\mathrm{Al}$ monowire, the Fermi point is at $1 / 4$ of the first BZ, so the Peierls longitudinal distortion should occur for a 4 atom unit cell. We verify using structural relaxation in this larger cell that the regularly spaced straight and obtuse angle structures are stable with respect to distortions, along the wire axis, and in all three directions, resp., confirming the phonon band structure (i.e. that the $\mathrm{X}$ point phonons are stable at equilibrium).

As the smearing scheme used for $\vec{k}$ integration has the
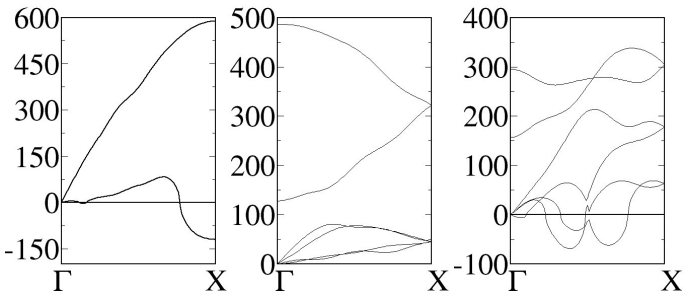

FIG. 4: Phonon band structure of different $\mathrm{Al}$ monowires, in $\mathrm{cm}^{-1}$. From left to right: 1-1, stretched zigzag obtuse, and stretched acute wires.
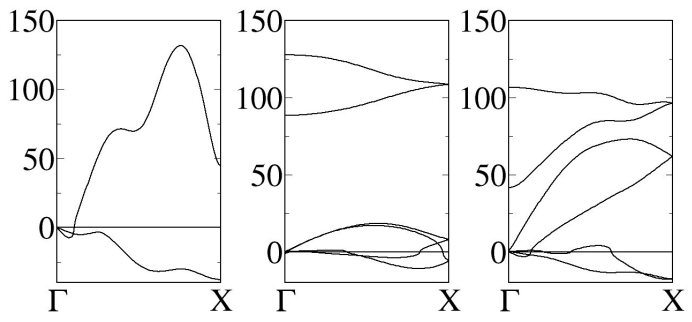

FIG. 5: Phonon band structure of different $\mathrm{Pb}$ monowires, in $\mathrm{cm}^{-1}$. From left to right: 1-1, obtuse, and acute wires.

same form as a temperature, this theoretical stability warrants a special note here: in 1D, the Fermi surface (FS) gives rise to systematic Kohn Anomalies (KA). The phonon frequencies near $\vec{q}=2 \overrightarrow{k_{F}}$ soften as $\mathrm{T} \rightarrow 0$. In the $\mathrm{Al}$ monowires the effect is very weak and quite localized in $\vec{q}$. For the $\mathrm{Pb}$ monowire the effect is very strong, and three bands cross the FS, giving rise to three $\vec{q}$ with KA. The strongest effect is at the $X$ point: the SO effect splits a degenerate band which (without SO) crosses the FS at reduced coordinate 0.18 . One band then crosses the FS at 0.1 and the other at 0.25 , exactly half of $X=0.5$. This is a coincidence which is specific to $\mathrm{Pb}$, not a resonant or stabilizing effect: decreasing the SO coupling moves the FS point away from 0.25 . We find that the same effect occurs in bulk FCC $\mathrm{Pb}$, with a softening of the $X$ point mode when SO is included, as was suggested in Ref. [25]. At $0 \mathrm{~K}$ the longitudinal phonon frequencies at $2 \overrightarrow{k_{F}}$ should diverge and become negative. We have not studied the renormalization of the phonon spectrum which would be needed to reproduce this effect analytically[5]. In both $\mathrm{Al}$ and $\mathrm{Pb}$ wires, however, reducing the smearing width to $0.1 \mathrm{mHa}(30 \mathrm{~K})$ leaves the longitudinal frequencies positive.

To describe the electron-phonon coupling in the nanowires, we examine the Eliashberg function $\alpha^{2} F$. The distorted $\mathrm{Pb}$ wires are not metallic, and their $\alpha^{2} F$ cannot be computed. The $\alpha^{2} F$ of the aluminum nanowires and lead monowire are shown in Fig. 6. For the Al monowire the coupling is extremely small. The transverse distortion to the $\mathrm{ZZ}$ structures (middle row) increases $\alpha^{2} F$ by several orders of magnitude. At this stage the EPC is comparable to that in bulk $\mathrm{Al}$, shown in the left middle panel of Fig. 6. Going on to the thicker wires shown 

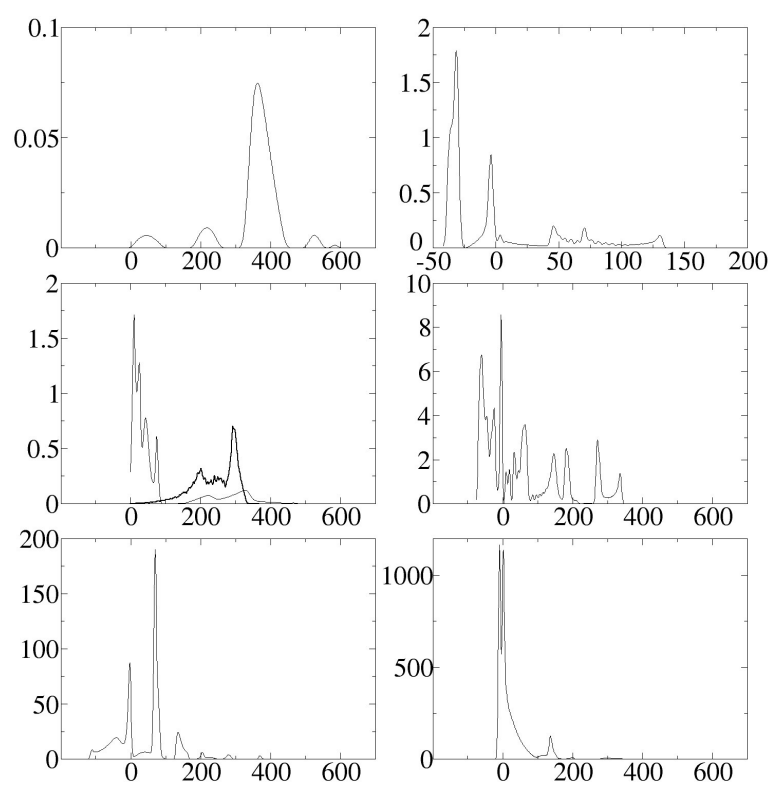

FIG. 6: $\alpha^{2} F$ as a function of frequency $\left(\mathrm{cm}^{-1}\right)$ for different nanowires. Top row: straight $\mathrm{Al}$ (left) and $\mathrm{Pb}$ (right). Middle: $\mathrm{Al} \mathrm{ZZ} \mathrm{obtuse} \mathrm{with} \mathrm{FCC} \mathrm{Al} \alpha^{2} F$ (thick line), and $\mathrm{ZZ}$ acute stretched wires. Bottom: Al 1-4 and 3-3 wires

in Fig. 1, the coupling continues to increase. The $\alpha^{2} F$ is very large around $\omega=0$ because $\alpha^{2} F$ varies as $1 / \omega$. The differences between the values of $\alpha^{2} F$ at low frequency for different wires reflect actual differences in the strength of the coupling, however, as they are in the same range of frequencies. The diverging $\alpha^{2} F$ would never be seen as the structures are unstable. The EPC will be smaller after relaxation as $\omega$ will be higher. The variations observed depend on the coordination with closely bonded neighbors. Very thick wires will contain bulk-like atoms and acquire the weak bulk EPC for Al. The EPC is strong in our thicker nanowires because all atoms are on the surface: the $1 \mathrm{D}$ characters of both the phonon and electron structures enhance the EPC. The $\mathrm{Pb}$ wire has a much stronger EPC than $\mathrm{Al}$, and also couples different modes to electrons. For Al the coupling to transverse modes is small $\left(\alpha^{2} F\right.$ less than 0.01), whereas for $\mathrm{Pb}$ most of the coupling is in the low and imaginary frequency transverse modes. We note that the preference of monowires to distort according to GPT is not dependent on the strength of the EPC or on the nature of the modes which couple: it appears to be a very general feature of nanowire structures.

To sum up the mechanism, in all the unstrained, fully relaxed, nanowires that we studied, unstable phonon modes are present. Strain can stabilize them in some cases. Moreover, the unstable modes are transverse and not longitudinal, in contrast with the usual Peierls mechanism. This implies that the successive relaxed structures conserve a plane of symmetry, and longitudinal pairing plays a minor role. The Peierls transitions are then gapless, and the system stays metallic. The longitudinal distortion remains energetically favorable at $0 \mathrm{~K}$ through the "normal" Peierls theorem, and at some point the wire will distort longitudinally, open a gap, and may stabilize. Importantly, the order in which the different transitions happen will be temperature dependent, as the normal Peierls transition depends on the softening of a longitudinal mode at $2 k_{F}$ with decreasing temperature. The electron phonon coupling is very different in $\mathrm{Al}$ and $\mathrm{Pb}$, but many common features remain. Finally, the spinorbit interaction in $\mathrm{Pb}$ leads to the opening of a gap in the zigzag wires.

The authors are grateful to P. Sen and X. Blase for stimulating discussions. This research has been supported by the Belgian FRFC (proj. 2.4502.05), the ARC "Interaction électron-vibration dans les nanostructures", the PAI/IUAP phase 5 "Quantum size effects in nanostructured materials", the NANOQUANTA EU Network of Excellence (NMP4-CT-2004-500198) and Marie Curie fellowship MEIF-CT-2005-024152.

* Electronic address: mjv500@york.ac.uk

[1] H. Fröhlich, Proc. R. Soc. London A 223, 296 (1954).

[2] R. Peierls, Quantum Theory of Solids (Oxford University Press, London, 1955), 1st ed.

[3] I.P. Batra, Phys. Rev. B 42, 9162 (1990).

[4] L. de la Vega, A. Martín-Rodero, N. Agrait, and A.L. Yeyati, Phys. Rev. B 73, 075428 (2006).

[5] D. Connétable, G.-M. Rignanese, J.-C. Charlier, and X. Blase, Phys. Rev. Lett. 94, 015503 (2005).

[6] K.P. Bohnen, R. Heid, H.J. Liu, and C.T. Chan, Phys. Rev. Lett. 93, 245501 (2004).

[7] H. Ohnishi, Y. Kondo, and K. Takayanagi, Nature 395, 780 (1998).

[8] A. Yanson, G. Bollinger, H. van den Brom, N. Agrait, and J. van Ruitenbeek, Nature 395, 783 (1998).

[9] J. Pascual, J. Méndez, J. Gómez-Herrero, A. Baró, N. Garcia, U. Landman, W.D. Luedtke, E. Bogachek, and H.-P. Cheng, Science 267, 1793 (1995).

[10] U. Landman, W.D. Luedtke, B.E. Salisbury, and R.L. Whetten, Phys. Rev. Lett. 77, 1362 (1996).

[11] D. Sanchez-Portal, E. Artacho, J. Junquera, P. Ordejon, A. Garcia, and J.M. Soler, Phys. Rev. Lett. 83, 3884 (1999).

[12] F.J. Ribeiro and M.L. Cohen, Phys. Rev. B 68, 035423 (2003).

[13] P. Sen, S. Ciraci, A. Buldum, and I.P. Batra, Phys. Rev. B 64, 195420 (2001).

[14] A. Ayuela, H. Räbiger, M.J. Puska, and R.M. Nieminen, Phys. Rev. B 66, 035417 (2002).

[15] T. Ono and K. Hirose, Phys. Rev. B 68, 045409 (2003).

[16] K.S. Thygesen and K.W. Jacobsen, Phys. Rev. Lett. 91, 146801 (2003).

[17] S. Michotte, L. Piraux, S. Dubois, F. Pailloux, G. Stenuit, and J. Govaerts, Physica C 377, 267 (2002).

[18] X. Gonze, J.-M. Beuken, R. Caracas, F. Detraux, M. Fuchs, G.-M. Rignanese, L. Sindic, M. Verstraete, G. Zerah, F. Jollet, M. Torrent, A. Roy, M. Mikami, Ph. Ghosez, J.-Y. Raty, D.C. Allan, Comp. Mat. Sci. 
25, 478 (2002).

[19] P. Hohenberg and W. Kohn, Phys. Rev. 136, 864 (1964)

[20] W. Kohn and L. Sham, Phys. Rev. 140, A 1133 (1965).

[21] N. Troullier and J.L. Martins, Phys. Rev. B 43, 1993 (1991).

[22] C. Hartwigsen, S. Goedecker, and J. Hutter, Phys. Rev. B 58, 3641 (1998)

[23] S. Baroni, S. de Gironcoli, A. Dal Corso, and P. Gian- nozzi, Rev. Mod. Phys. 73, 515 (2001).

[24] P. B. Allen and B. Mitrović, Theory of Superconducting $T_{c}$, vol. 37 of Solid State Phys. (Academic Press, New York, 1982).

[25] A.Y. Liu and A.A. Quong, Phys. Rev. B 53, R7575 (1996). 